\title{
Moagem de pós de carbeto de silício em moinho planetário
}

\section{(Planetary milling of silicon carbide powders)}

\author{
M. A. P. dos Santos ${ }^{1}$, C. A. Costa ${ }^{2}$ \\ ${ }^{I}$ Grupo de Materiais, Instituto de Pesquisas da Marinha - IPqM, R. Ipirú s/n, Ilha do Governador \\ Rio de Janeiro, RJ 21931-090 \\ ${ }^{2}$ Programa de Engenharia Metalúrgica e de Materiais, COPPE - UFRJ, CP 68505 \\ Rio de Janeiro, RJ 21945-970
}

\begin{abstract}
Resumo
Pó de alfa carbeto de silício $(\alpha-\mathrm{SiC})$ foi cominuído em moinho planetário durante os períodos de tempo de $1 / 2,2,4$ e $6 \mathrm{~h}$. A velocidade de rotação foi de $300 \mathrm{rpm}$, o meio de moagem foi álcool isopropílico e os corpos moedores foram esferas de zircônia estabilizada com céria. Os pós cominuídos foram caracterizados quanto ao tamanho e distribuição de tamanho de partícula, a composição química, as fases cristalinas e a morfologia. Observou-se uma grande redução no tamanho de partículas, que passaram de micrométricas para submicrométricas e até nanométricas, alteração da distribuição das mesmas de bimodal para unimodal, e a geração de aditivação secundária causada pelo meio de moagem.

Palavras-chave: moagem, nanopós, SiC, aditivação secundária.
\end{abstract}

\begin{abstract}
Alpha silicon carbide $(\alpha-S i C)$ powder was comminuted in planetary mill during 1/2, 2, 4 and 6 h. The rotation speed was 300 rpm, the medium of milling was isopropyl alcohol and the grinding bodies were spheres of ceria-stabilized zirconia. The milling powders were characterized concerning the size and particle size distribution, the chemical composition, the crystalline phases, and morphology. A large reduction of the particle size, that had changed from micrometrics to submicrometrics and even nanometrics, a change of their distribution from bimodal to unimodal, and the generation of secondary aditivation caused by medium of milling were observed.
\end{abstract}

Keywords: milling, nanopowders, SiC, secondary activation.

\section{INTRODUÇÃO}

As propriedades de um produto cerâmico são dependentes das matérias primas utilizadas, do método empregado no processamento do corpo a verde, do tipo e das condições de sinterização. As matérias-primas altamente covalentes, como carbetos e nitretos, possuem sinterabilidade inerentemente baixa, decorrente da alta energia da ligação atômica. Uma das maneiras mais eficientes de se aumentar a sinterabilidade é por meio da redução do tamanho de partícula, cujos efeitos terão forte influência sobre as demais etapas do processamento e nas propriedades finais do material $[1,2]$.

Uma grande variedade de métodos físicos, químicos e mecânicos são disponíveis para a preparação de pós finos e ultrafinos [3]. Os métodos mecânicos que produzem pós submicrométricos e nanométricos são comumente classificados de moagem de alta energia e alguns equipamentos típicos empregados são moinho a jato, planetário e atritor. Esses processos têm sido utilizados tanto em escala laboratorial como industrial [3, 4].
Resumidamente, o processo de moagem a jato ocorre através da colisão entre partículas impulsionadas por um jato de gás sob alta pressão. Este se dá a seco, a contaminação é nula, baixo níveis de desgaste do equipamento e de ruído, sendo estas as grandes vantagens do processo. Todavia, a perda de produto é elevada, necessita de um sistema classificação de alta qualidade, o custo do equipamento é elevado e problemas de reaglomeração devido a cargas eletrostáticas estão sempre presentes [4-7]. Já a moagem, tanto em moinho planetário como em atritor, ocorre através do choque mecânico entre os corpos moedores e as partículas. Normalmente o processo se dá em meio úmido, possui elevado desgaste do equipamento e o grau de contaminação é bastante dependente do meio de moagem empregado, isto é, quando mais próxima de uma condição autógena, menor a contaminação. A perda de material é pequena e o custo do equipamento é significantemente menor, especialmente quando comparada com a moagem a jato, a homogeneização é muito boa e partículas com elevada área superficial são facilmente geradas em um tempo muito curto [8-11]. 
Estudos de moagem de carbeto de silício utilizando moinho a jato, planetário e atritor não são encontrados com facilidade na literatura aberta. Dados do ISI webofscience mostraram a publicação de aproximadamente 300 artigos desde 1960 sobre moagem de alta energia, mas apenas um envolvendo carbeto de silício. Desta forma, há uma lacuna a ser preenchida no que toca a moagem de $\mathrm{SiC}$ utilizando moagem de alta energia. E, no sentido de suprir esta lacuna, realizou-se este trabalho em moinho planetário cujos parâmetros envolvidos foram a influência do tempo de moagem sobre a distribuição, tamanho, morfologia e contaminação dos pós moídos. O resultado final mostra que o processo gera pós submicrométricos com estreita distribuição de tamanho de partícula, além de possuírem elevada sinterabilidade.

\section{MATERIAIS E MÉTODOS}

Utilizou-se como matéria prima o carbeto de silício SiC1000 (Alcoa Alumínio S.A., Brasil). A caracterização do pó mostrou que o mesmo apresenta fase cristalina alfa $(\alpha-\mathrm{SiC})$, densidade $3,211 \mathrm{~g} / \mathrm{cm}^{3}$ e distribuição bimodal de tamanho de partícula, sendo os valores de $\mathrm{d}_{99}$ e $\mathrm{d}_{50}$ iguais a 14,22 e $1,77 \mu \mathrm{m}$, respectivamente, enquanto a composição química é mostrada na Tabela I [12].

$\mathrm{O}$ processo de moagem foi realizado em moinho planetário (modelo PM-4, Retsch). Os jarros utilizados na moagem foram fabricados especialmente para suportar os elevados desgastes do processo e moer quantidades de material superiores a $100 \mathrm{~g}$ por jarro. Eles foram confeccionados em aço inoxidável, com $10 \mathrm{~cm}$ de diâmetro interno, capacidade de $500 \mathrm{~mL}$, e revestidos internamente com carbeto de tungstênio (WC-Co) pelo processo HVOF (High Velocity Oxygen Fuel). As condições de moagem foram: velocidade de rotação de $300 \mathrm{rpm}$, álcool isopropílico P.A, corpos moedores de zircônia estabilizada com céria $\left(80 \% \mathrm{ZrO}_{2}+20 \% \mathrm{CeO}_{2}-\mathrm{Zirconox}{ }^{\circledR}\right.$, Netzsch), cuja faixa granulométrica variou de 0,7 a $1,2 \mathrm{~mm}$ de diâmetro, densidade $6,1 \mathrm{~g} / \mathrm{cm}^{3}$, e dureza Vickers $1200 \mathrm{kgf} / \mathrm{mm}^{2}$.

Os jarros de moagem foram preenchidos pela metade $(250 \mathrm{~mL})$ e cerca de $60 \%$ deste volume $(150 \mathrm{~mL})$ foram preenchidos pelos corpos moedores, enquanto os $40 \%$

Tabela I - Composição química do pó como recebido [12]. [Table I - Chemical composition of the as received powder].

\begin{tabular}{cc}
\hline Composição & peso \% \\
\hline $\mathrm{SiC}$ & 98,71 \\
$\mathrm{Si}+\mathrm{SiO}_{2}$ & 0,60 \\
$\mathrm{Fe}$ & 0,30 \\
$\mathrm{Al}$ & 0,19 \\
Carbono Livre (CL) & 0,20 \\
$\mathrm{~S}, \mathrm{Ca}, \mathrm{Ti}, \mathrm{V}, \mathrm{Ni}, \mathrm{Cu}$ e Zr & Traço \\
\hline
\end{tabular}

restantes $(100 \mathrm{~mL})$ foram compostos pela polpa, na seguinte proporção: $100 \mathrm{~g}$ de $\mathrm{SiC}$ e álcool isopropílico em volume suficiente para cobrir o pó de $\mathrm{SiC}$ e os corpos moedores.

Os tempos de moagem foram de $1 / 2,2,4$ e $6 \mathrm{~h}$. Após as moagens, os pós foram colocados em bandejas pirex ${ }^{\circledR}$, secos em estufa a $70^{\circ} \mathrm{C}$ por $24 \mathrm{~h}$ e em seguida desaglomerados em gral de ágata.

A caracterização dos pós moídos, com respeito à distribuição e tamanho médio de partícula, foi feita em equipamento MAF 5001 (Malvern Mastersizer Micro Plus), que tem capacidade de medir partículas nas faixas de 0,05 até $500 \mu \mathrm{m}$. A área superficial específica foi medida pela técnica de fisisorção (Gemini III 2375, Micromeritics), enquanto a determinação da composição química foi feita com espectrômetro de fluorescência de raios X (Philips PW 2400 com tubo de Rh) e difração de raios X (Siemens modelo D 5005). A morfologia das partículas foi observada com o auxílio de microscópio eletrônico de varredura (Leica Stereoscan, modelo S 440 ).

\section{RESULTADOS E DISCUSSÃO}

A obtenção de elevada densidade em materiais cerâmicos tem como um dos principais requisitos que os pós de partida sejam finos (pequeno tamanho de partícula) e possuam estreita distribuição granulométrica. Quando processos de moagem de alta energia são empregados para este fim, deve-se atentar para o grau de contaminação introduzido pelo mesmo, o qual normalmente é função do meio e do tempo de moagem [13]. A relação entre estes três itens será mostrada abaixo para o presente estudo da moagem do carbeto de silício.

A influência do tempo de moagem sobre a redução do tamanho médio $\left(\mathrm{d}_{50}\right)$ de partícula do $\alpha$-SiC pode ser observada na Tabela II, frisando que as condições de moagem foram constantes: velocidade $300 \mathrm{rpm}$, corpos moedores de $\mathrm{ZrO}_{2}: \mathrm{CeO}_{2}$ e meio de moagem álcool isopropílico P.A.. Observa-se que $50 \%$ das partículas atingem rapidamente um limite de saturação de aproximadamente $0,4 \mu \mathrm{m}$. Estes dados indicam que o processo usado foi extremamente eficiente em reduzir o tamanho de partícula em curtos espaços de tempo de moagem e que tempos mais longos

Tabela II - Tamanho médio de partícula $\left(\mathrm{d}_{50}\right)$ em função do tempo de moagem em álcool isopropílico.

[Table II - Average particle size $\left(d_{50}\right)$ as a function of the milling time in isopropyl alcohol.]

\begin{tabular}{cc}
\hline $\begin{array}{c}\text { Tempo de Moagem } \\
(\mathrm{h})\end{array}$ & $\begin{array}{c}\text { Tamanho de Partícula } \mathrm{d}_{50} \\
(\mu \mathrm{m})\end{array}$ \\
\hline Original $(0)$ & 1,77 \\
$1 / 2$ & 0,41 \\
2 & 0,38 \\
4 & 0,36 \\
6 & 0,36 \\
\hline
\end{tabular}


(\%)

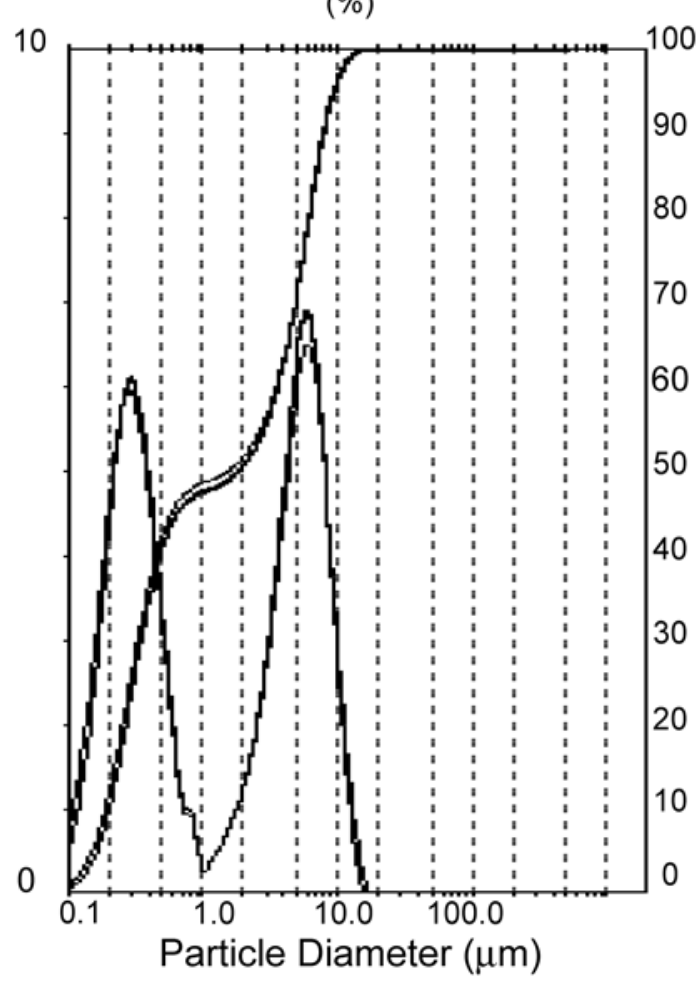

(a)
(\%)

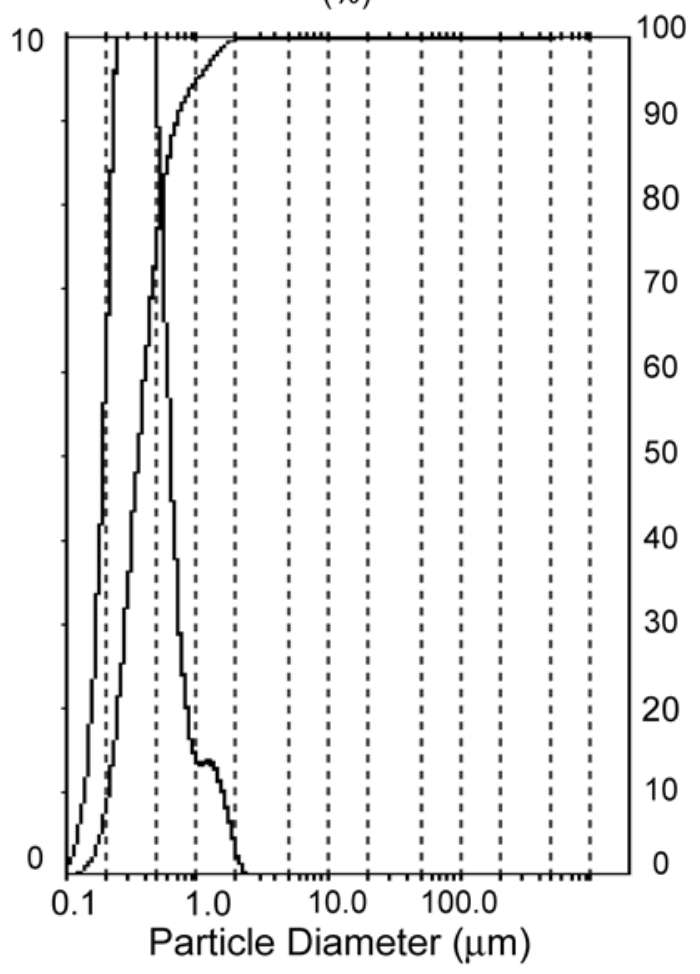

(b)

Figura 1: Distribuição bimodal do pó de SiC como recebido (a) e distribuição unimodal do pó de SiC cominuido por 6 h (b).

[Figure 1: Bimodal distribution of the as-received SiC powder (a) and unimodal distribution of the SiC powder after milling for $6 \mathrm{~h}$ (b).]

estão associados, majoritariamente, a quebra de partículas grosseiras. Para fins comparativos, valores típicos de $d_{50}$ de pós de $\mathrm{SiC}$ comercialmente disponíveis no mercado estão na faixa 3,0 a 1,8 $\mu \mathrm{m}[4,12]$, o que torna o material moído propício para sinterização. Variações no meio de moagem, como já usado [13], velocidade de rotação e/ou dimensão dos corpos moedores podem melhorar o processo, mas estudos confirmativos precisam ser feitos.

Com respeito a distribuição do tamanho de partícula, observou-se que o pó como recebido e os cominuídos por $1 / 2,2$ e $4 \mathrm{~h}$ apresentaram comportamento bimodal, enquanto o cominuído por $6 \mathrm{~h}$ mostrou comportamento unimodal, conforme exemplificado na Fig. 1. O pó como recebido (Fig. 1a) possui os dois picos da distribuição centrados em 0,2 e $5 \mu \mathrm{m}$, enquanto o pó moído por $6 \mathrm{~h}$ (Fig. $1 \mathrm{~b}$ ) teve seu único pico centrado em $0,2 \mu \mathrm{m}$. Os gráficos de distribuição de tamanho de partícula para os vários tempos de moagem confirmam que a redução do tamanho das partícula se dá unicamente pela quebra das partículas maiores, uma vez que o pico de 0,2 $\mu \mathrm{m}$ mantém-se fixo em todos eles. Este valor sugere que há um limite abaixo do qual não é mais possível reduzir o tamanho das partículas com as condições de moagem usadas.

O processo de moagem empregado neste trabalho mostrou-se altamente eficiente em produzir partículas submicrométricas e até nanométricas. Contudo, faz-se necessário verificar se a redução de tamanho de partícula foi acompanhada pela introdução de outros compostos/

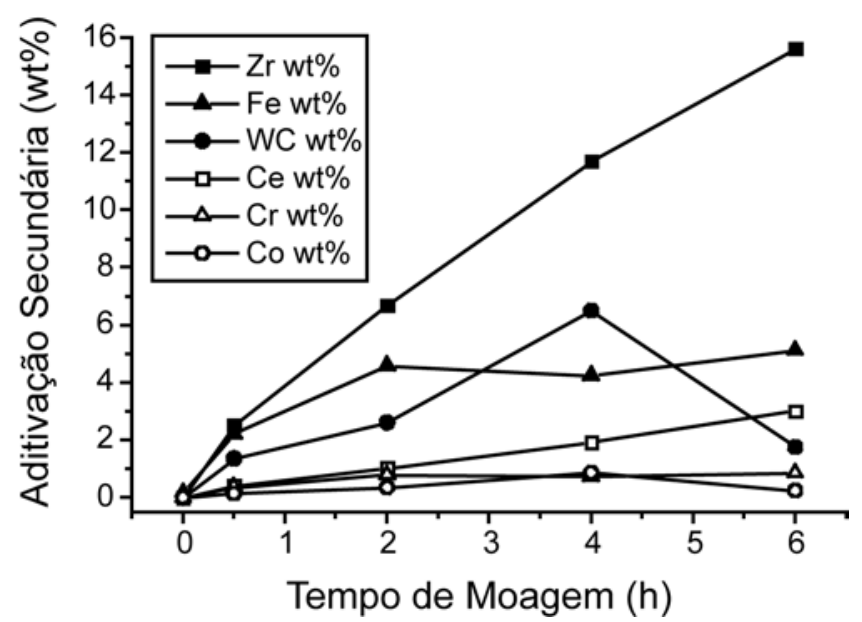

Figura 2: Quantificação dos elementos introduzidos pelo processo de moagem em função do tempo moagem do $\alpha$-SiC. Técnica: fluorescência de raios X.

[Figure 2: The quantification of the elements introduced by the milling process as a function of the milling time for $\alpha-S i C$. X-ray fluorescence technique.] 


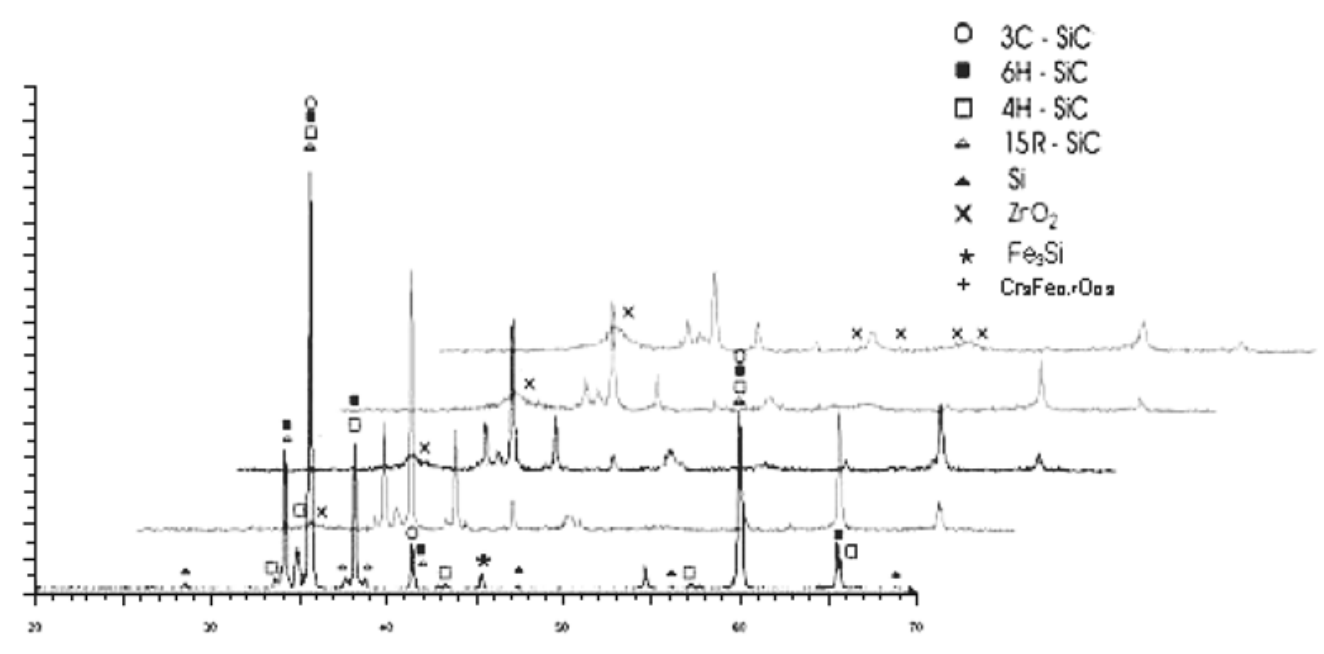

Figura 3: Difratogramas do pó de $\mathrm{SiC}$ como recebido e resultantes de todos os tempos de moagem. O primeiro difratograma corresponde ao pó como recebido, o segundo ao pó moído por $1 / 2 \mathrm{~h}$ e os demais, em ordem consecutiva, aos tempos de moagem de 2,4 e $6 \mathrm{~h}$.

[Figure 3: X-ray diffraction patterns of the as-received SiC powders and from all the milling times, in consecutive order.]

elementos provenientes da elevada abrasividade do processo de moagem. Para tal fim, utilizou-se a combinação das técnicas de fluorescência e de difração de raios X, pois identificam elementos químicos e fases cristalinas, respectivamente. A caracterização por fluorescência de raios $X$ (Fig. 2) mostrou que houve elevada aditivação secundária em relação ao SiC. O termo aditivação secundária será usado ao invés de contaminação, visto que esta não prejudicou a sinterização [12] e melhorou a tenacidade à fratura do material sinterizado [14].

A maior aditivação foi de $\mathrm{ZrO}_{2}$, que aumentou continuamente com o tempo de moagem, atingindo valores da ordem de $15 \%$ em peso para o tempo de $6 \mathrm{~h}$. Em seguida, o $\mathrm{Fe}$, teve seu valor elevado para aproximadamente $5 \%$ em peso após $2 \mathrm{~h}$ de moagem e, doravante, se manteve praticamente constante. $\mathrm{O}$ teor de $\mathrm{WC}$ aumentou de forma descontínua até $6,5 \%$ em peso para $4 \mathrm{~h}$ de moagem e, posteriormente, teve seu valor reduzido para $2 \%$ em peso. Outros compostos que aditivaram as amostras foram o $\mathrm{CeO}_{2}$, Co e Cr, como era de se esperar, visto que esses elementos já faziam parte das esferas de moagem $\left(80 \% \mathrm{ZrO}_{2}+20 \%\right.$ $\mathrm{CeO}_{2}$ ), do recobrimento (WC+Co) aplicado por $\mathrm{HVOF}$ e do aço inox, respectivamente. As curvas de comportamento do $\mathrm{CeO}_{2}$, $\mathrm{Co}$ e $\mathrm{Cr}$ foram similares às observadas do elemento parente, isto é, as curvas de $\mathrm{Zr}$ e Ce subiram continuamente, enquanto as do WC e Co atingiram um máximo em $4 \mathrm{~h}$ de moagem e depois diminuíram, e as de $\mathrm{Fe}$ e $\mathrm{Cr}$ alcançaram o máximo em $2 \mathrm{~h}$ de moagem e permaneceram praticamente constantes.

O comportamento regular dos elementos permite a seguinte explicação: no início da moagem (até 2 h) os teores dos compostos/elementos aumentam continuamente em função do forte atrito entre as esferas de moagem, o pó, o revestimento e pequenas regiões da parede sem revestimento, que resulta na introdução contínua de pequenos fragmentos oriundos de cada um destes locais. Para tempos superiores a $2 \mathrm{~h}$, cada região necessita ser avaliada isoladamente. $\mathrm{O}$ contínuo aumento dos teores de $\mathrm{ZrO}_{2}$ e $\mathrm{CeO}_{2}$ é proveniente das esferas de moagem que por serem excessivamente finas estão sujeitas a uma maior taxa de desgaste abrasivo, devido à sua maior área superficial; todavia, estas também causam desgaste do revestimento interno $(\mathrm{WC}+\mathrm{Co})$ dos vasos de moagem. O comportamento do WC e Co sugere a existência de dois efeitos combinados, quais sejam: i) o revestimento de $\mathrm{WC}+\mathrm{Co}$ passaria a ter uma camada superficial de pós muito finos de $\mathrm{SiC}$ ou de zircônia cominuídos e, assim, impedindo novos choques e atritos dos corpos moedores com o revestimento, e ii) observouse que o revestimento tornava-se mais e mais polido conforme o tempo de moagem aumentava, diminuindo progressivamente a rugosidade da superfície. Com respeito a contaminação com $\mathrm{Fe}$ e $\mathrm{Cr}$ as seguintes colocações podem ser feitas: o $\alpha$-SiC como recebido apresentou um percentual de $\mathrm{Fe}$ de 0,30 peso\%, porém sua quantidade após $2 \mathrm{~h}$ de moagem ficou constante em aproximadamente $5 \%$. Provavelmente, esta enorme contaminação pode ser devida a pequenas fendas que não foram completamente revestidas com WC+Co durante a aplicação com HVOF. Neste caso, o contato do pó, extremamente abrasivo, com o aço inoxidável macio, resultaria na contaminação tanto pelo Fe como pelo Cr. Coincidentemente, ambos os elementos apresentam comportamento idênticos durante a moagem, isto é, elevam os seus teores até $2 \mathrm{~h}$ e depois permanecem constantes, possivelmente, devido a deposição de uma camada protetora superficial de $\mathrm{SiC}$, tal como proposto para o revestimento.

$\mathrm{A}$ análise de difração de raios $\mathrm{X}$ do pó como recebido e dos cominuídos estão mostrados na Fig. 3.Observa-se que o pó recebido apresenta os picos correspondentes aos polítipos 
3C, $6 \mathrm{H}, 4 \mathrm{H}$ e 15R, típicos do $\alpha-\mathrm{SiC}$ [15]. Conforme o pó foi cominuído até $6 \mathrm{~h}$, observa-se gradativo aumento da intensidade dos picos de $\mathrm{ZrO}_{2}$ desde $1 / 2$ até $6 \mathrm{~h}$ de moagem, tal como observado na análise de fluorescência. Além disso, foram identificados picos referentes ao $\mathrm{Fe}$, cujo teor em peso no pó como recebido é de $0,30 \%$. Percebe-se também que as intensidades dos picos de $\mathrm{SiC}$ diminuem com o tempo de moagem, o que sugere, que a porção de partículas maiores, responsáveis por picos finos e mais altos [12], é cada vez menor. Este tipo de observação foi mais nítida para os tempos de moagem de 4 e $6 \mathrm{~h}$, quando as partículas analisadas possuem dimensões submicrométricas e nanométricas. Estes dados indicam que o efeito de aglomeração de partículas finas, pode estar acontecendo, uma vez que tanto a diminuição da intensidade como a abertura dos picos de raios X estão correlacionados com dimensões nanométricas [16-19].

Tão importante quanto à redução do tamanho de partícula é observar se houve alteração da geometria das mesmas. Neste sentido, análise por microscopia eletrônica de varredura foi realizada nos pós após os tempos de moagem. As Figs. 4 e 5 mostram o pó como recebido e o moído por $6 \mathrm{~h}$, respectivamente. $\mathrm{Na}$ Fig. 4, observam-se partículas não esféricas, várias faces marcadas por arestas vivas e não há aglomeração, enquanto a Fig. 5, com o mesmo aumento da Fig. 4, mostra a grande redução sofrida pelas mesmas e a formação de aglomerados. $\mathrm{Na}$ observação com maior aumento, do pó moído por 6 h (Fig. 6), notou-se que as partículas passaram a ter uma morfologia bem mais arredondada, além da constatação da presença de $\mathrm{ZrO}_{2}$ nas mesmas, identificadas como partículas de cor clara (branca).

Os dados acima mostraram que a cominuição em moinho planetário foi muito eficiente em reduzir as partículas de carbeto de silício em curto espaço de tempo, apesar da

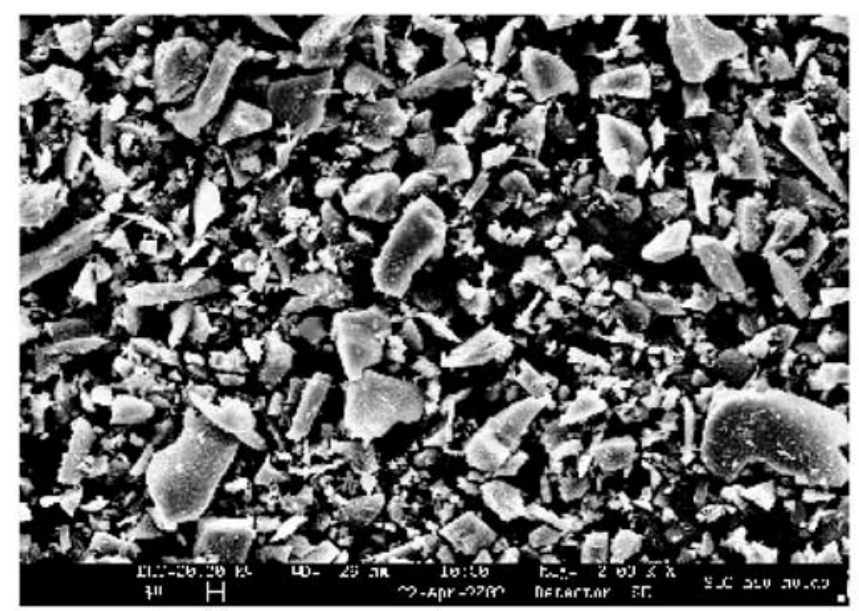

Figura 4: Fotomicrografia do $\alpha$-SiC como recebido. O tamanho médio de partícula $\left(\mathrm{d}_{50}\right)$ é $1,77 \mu \mathrm{m}$. Notam-se partículas não esféricas e várias faces marcadas por arestas vivas. Aumento de $2 \mathrm{k}$ X.

[Figure 4: SEM photomicrograph of the as-received $\alpha$-SiC powder. The average particle size $\left(d_{50)}\right.$ was $1.77 \mu \mathrm{m}$ and morphology showed marked sharp edges.]

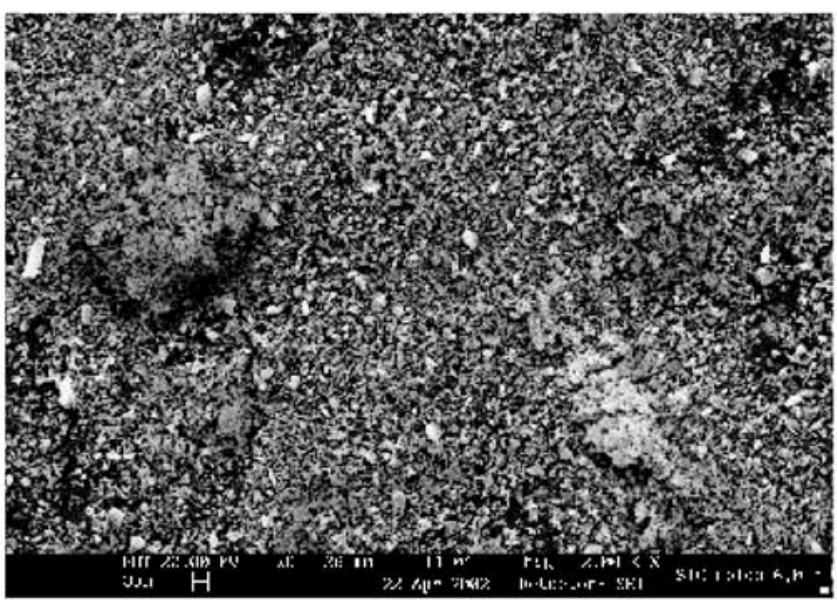

Figura 5: Pó $\alpha$-SiC resultante de 6 h de moagem, com tamanho médio de partícula $0,38 \mu \mathrm{m}$ e aspecto bastante aglomerado. Aumento de $2 \mathrm{k}$ X.

[Figure 5: SEM photomicrograph of $\alpha$-SiC powder milled for $6 \mathrm{~h}$ in planetary mill, showing an average particle size of $0.38 \mu \mathrm{m}$ and in a very agglomerated form.]

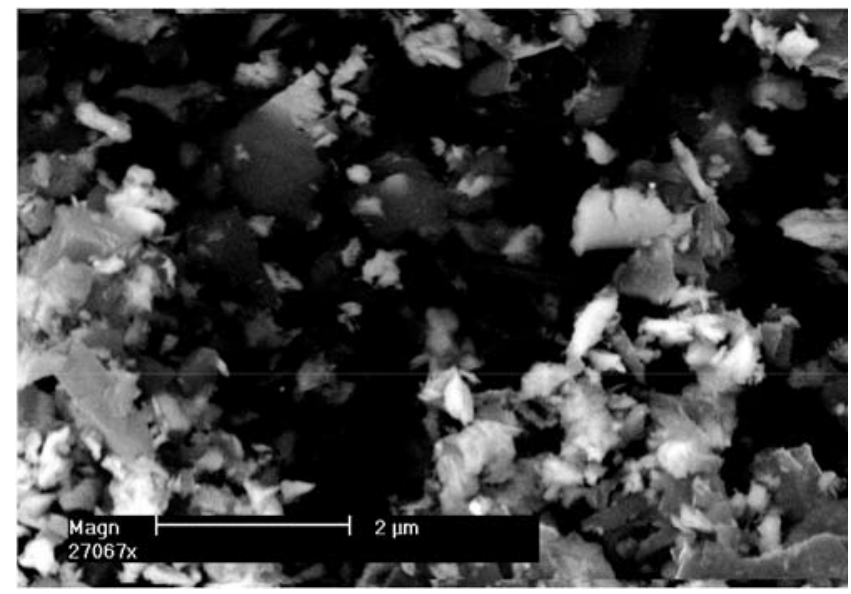

Figura 6: Magnificação da Fig. 5, mostrando que o pó possui partículas bem arredondadas, sem arestas vivas. As partículas brancas foram caracterizadas como $\mathrm{ZrO}_{2}$. Aumento de $2 \mathrm{k} \mathrm{X}$. [Figure 6: Higher magnification of Fig. 5, showing very agglomerated particles, with rounded off edges. The white particles were characterized as $\mathrm{ZrO}_{2}$.]

contaminação secundária introduzida. Na análise de tamanho de partícula, considerou-se a presença única de $\mathrm{SiC}$, mas cabe ressaltar a presença de partículas provenientes da aditivação secundária nesta análise, as quais devem ter a mesma ordem de grandeza ou serem ainda menores. Esta constatação pode ser tirada pelo fato da distribuição de partículas ter ocorrido de forma a torná-la unimodal e centrada nas partículas menores (com tamanho de $0,2 \mu \mathrm{m}$ ). A separação do $\mathrm{SiC}$ dos demais elementos, sob a pura ótica de análise de moagem, a qual pode ser feita através de decantação, seria muito interessante para que a eficiência do processo pudesse ser 
avaliada com parâmetros reais.

Do ponto de vista de efeito da alteração química sobre a sinterabilidade do $\mathrm{SiC}$, cabe citar que a presença de aditivos pode beneficiar o processo de sinterização, sendo o caso mais típico a adição de carbono e boro na sinterização do $\mathrm{SiC}$ [20-22] e de $\mathrm{ZrO}_{2}$ melhorando a sinterabilidade do $\mathrm{B}_{4} \mathrm{C}$ através da formação de $\mathrm{ZrB}$ [23]. Como os trabalhos termodinâmicos de Negita [24] colocam a $\mathrm{ZrO}_{2}$ próximo ao campo de sinterabilidade do $\mathrm{SiC}$ e como não foi encontrado na literatura situação similar a esta, procedeu-se os estudos de sinterabilidade e tenacidade à fratura dos presentes materiais. Os resultados mostraram que a aditivação secundária não prejudicou a sinterização e foi benéfica com a tenacidade à fratura, pois materiais com densidade de $99 \%$ e $\mathrm{K}_{\mathrm{IC}}$ de 5,5 MPa.m ${ }^{1 / 2}$ foram obtidos [12, 14].

\section{CONCLUSÃO}

A moagem de $\mathrm{SiC}$ em moinho planetário, utilizando-se álcool isopropílico, resultou em grande e rápida redução do tamanho de partícula, com $\mathrm{d}_{50}$ de $0,4 \mu \mathrm{m}$ em apenas $30 \mathrm{~min}$ de moagem. Paralelamente, teores de $\mathrm{ZrO}_{2}, \mathrm{CeO}_{2}$ e Fe aumentam continuamente com o tempo de moagem até $6 \mathrm{~h}$, atingindo valores de até $15 \%$ para $\mathrm{ZrO}_{2}$. Já o composto WC-Co aumentou até $4 \mathrm{~h}$ de moagem e, então, sofreu redução drástica. Esta queda observada no WC-Co está provavelmente associada com o polimento das paredes do vaso (redução de rugosidade) e ao estabelecimento de uma camada fina de $\mathrm{SiC}$ que impede novos contatos diretos entre as bolas de moagem e a parede. A substituição de esferas pesadas de $\mathrm{ZrO}_{2}$ por esferas leves de $\mathrm{SiC}$ talvez altere a eficiência de moagem, mas com certeza irá reduzir a contaminação final, tornando o material mais adequado para sinterização em estado sólido.

\section{REFERÊNCIAS}

[1] A. G. P. Silva, C. A. Júnior, "Teoria de sinterização por fase sólida; uma análise crítica de sua aplicação", Cerâmica 44, 286 (1998).

[2] S. T. Buljan, A. E. Pasto, H. J. Kim, "Ceramic whisker and particulate composites: properties, reliability and applications", Am Ceram. Soc. Bull. 68, 2 (1989) 387-394. [3] V. S. Aranachalan, R. Sundaresan, Powder Metallurgy, pp.139-192, in Processing of metals and alloys, Ed. R. W. Cahn, Vol.151 (1991).

[4] X.-Z. Guo, H. Yang, "Investigation of compaction and sintering behavior of $\mathrm{SiC}$ powder after ultra-fine treatment", http://www.zju.edu.cn/izus/preview/2003-B-53.doc.

[5] H. W. Hennicke, J. Stein, "Process of fine milling for ceramic materials", Mater. Sci. \& Eng. A 109 (1989) 3-7

[6] H. Berthiaux, J. Dodds, "Modelling fine grinding in a fluidized bed opposed jet mill - Part I: batch grinding kinetics", Powder Technol. 106 (1999) 78-87.

[7] A. C. Bentham, C. C. Kwan, R. Boerefijn, M. Ghadiri, "Fluidised-bed jet mill of pharmaceutical powders", Powder
Technol. (2004) in press

[8] H. Mio, J. Kano, F. Saito, K. Kaneko, "Effects of rotational direction and rotation-to-revolution speed ratio in planetary ball milling, Mater. Sci. \& Eng. A 332 (2002) 75-80.

[9] K. Shinohara, B. Golman, T. Uchiyama, M. Otani, "Finegrinding characteristics of hard materials by attrition mill", Powder Technol. 103 (1999) 292-296

[10] R. J. Rajamani, P. Songfack, B. K. Mishra, "Impact energy spectra of tumbling mills", Powder Technol. 108 (2000) 116-121.

[11] R. Hogg, A. J. Dynys, H. Cho "Fine grinding of aggregated powders", Powder Technol. 122 (2002) 122-128.

[12] M. A. P. Santos, "Processamento e sinterização de carbeto de silício nacional", Tese de Doutorado, PEMM, COPPE, UFRJ (2003).

[13] B. B. Matos, "Influência do meio na moagem ultrafina de carbeto de silício", Projeto de Formatura, Departamento de Engenharia Metalúrgica e de Materiais, Escola de Engenharia, Universidade Federal do Rio de Janeiro, RJ (2002).

[14] E. S. Lima, L. P. Brandão, C. A. Costa, "Mechanical behavior of a-SiC-based nanocomposites", II Encontro da SBPMat, Rio de Janeiro, RJ (2003).

[15] P. E. Praes, M. M. da Silva, "Uma revisão da síntese de pós cerâmicos via alcóxidos - estudos de caso: alcóxidos de terras-raras", Série Tecnologia Mineral, n. 71, CETEM/ CNPQ, Rio de Janeiro (1995).

[16]H.P. Klug, L. E. Alexander, X-ray diffraction procedures, John Wiley and Sons, New York, (1974) pp. 643.

[17] C. N. J. Wagner, E. N. Aqua, Advances in X-ray analysis, Plenum Press, New York, 7 (1964) pp. 88-96.

[18] H. G. Jiang, M. Ruhle, E. J. Laverning, "On the applicability of the X-ray diffraction line profile analysis in extracting grain size and microstrain in nanocrystalline materials", J. Mater. Res. 14, 2 (1999) 549-559.

[19] C. Suryanarayana, M. G. Norton, "X-ray diffraction: a practical approach", D. L. Dorset, Plenum Press, New York, (1998) p. 207.

[20] H.-W. Kim, Y.-H. Koh, H.-E. Kim, "Densification and mechanical properties of $\mathrm{B}_{4} \mathrm{C}$ with $\mathrm{Al}_{2} \mathrm{O}_{3}$ as a sintering aid", J. Am. Ceram. Soc. 83, 11 (2000) 2863-2865.

[21] X. F. Zhang, Q. Yang, L. C. De Jonghe, "Microstructure development in hot-pressed silicon carbide : effects of aluminum, boron, and carbon additives", Acta Mater. 51, 13 (2003) 3849-3860.

[22] Y. Zhou, H. Tanaka, S. Otani, Y. Bando, "Low temperature pressureless sintering of alpha-SiC with $\mathrm{Al}_{4} \mathrm{C}_{3}$ $\mathrm{B}_{4} \mathrm{C}$ additions", J. Am. Ceram. Soc. 82, 8 (1999) 1959.

[23] G. Magnani, G. Beltrami, "Pressureless sintering and properties of alpha-SiC-B ${ }_{4}$ C composite", J. Eur. Ceram. Soc. 21, (2001). 633-638

[24] K. Negita, "Effective sintering aids for silicon carbide ceramics: reactivities for silicon carbide with various additives", J. Am. Ceram. Soc. 69, (1986) 308-310.

(Rec. 22/04/2005, Ac. 26/05/2005) 